\title{
GLOBALIZACIÓN, EMPRESA Y ESTADO DE DERECHO EN EL PERÚ
}

\author{
Maximo Ugarte Vega Centeno* \\ mugartev@yahoo.com
}

\begin{abstract}
RESUMEN
La globalización viene a ser una etapa de la internacionalización de la economía que trae como consecuencia una interdependencia en el mercado por parte de las empresas, los países y las garantías legales de las personas para realizar sus actividades económicas. Hoy en día las empresas tienen que afrontar este fenómeno y a asumir retos tanto en su entorno interno como externo, y adecuarse a las exigencias de los nuevos consumidores en un estado derecho.
\end{abstract}

Palabras clave: Globalización, Empresa y Estado de Derecho.

\section{ABSTRACT}

The globalization brought us the internalization of the economy, in consequence the interdependency in the market, between countries, businesses and legal warranties for people who want to do economic activities. Nowaday the businesses have to face this phenomenon and take on the challenge as much internal as external environment. Also they have to adapt to the demands of the new consumers in the rule of Law.

Keywords: Globalization, Business and Rule of Law.

* Diplomado en Gestión por el Programa de Alta Dirección (PAD), Universidad Privada de Piura; Magíster por la Universidad de Barcelona (España) y Doctor en Estudios Internacionales por la Universidad de Córdoba (España). Profesor Principal de la Universidad Nacional mayor de San Marcos. 


\section{INTRODUCCIÓN}

Asistimos a un proceso creciente de globalización caracterizado, principalmente, por la información, el conocimiento y la tecnología que serán compartidos ampliamente y por los que estaremos cada vez más interconectados. En esa orientación dicho fenómeno global ha traído consecuencias en la inversión en el ámbito de la empresa y el Estado de Derecho.

En las empresas, las consecuencias más inmediatas han sido cómo afrontar su entorno interno y externo. En el ámbito interno existen retos pendientes de cambiar, partiendo del pensamiento empresarial que tuvo que adaptarse a los nuevos tiempos y pasando por los cambios internos que toda empresa tiene que realizar si pretende ser competitiva en este mercado global.

En el ámbito externo, las empresas tienen que seguir asumiendo los retos económicocomerciales, los vinculados con la tecnología y competitividad, el nuevo rol de los consumidores y el Estado de Derecho como garantía para las actividades económicas que deseen realizar las personas naturales y jurídicas y que nuestro ordenamiento jurídico nacional les brinda.

Así mismo, entendiendo que la globalización abarca también los procesos políticos, económi$\cos$, sociales, y culturales que se vienen suscitando a nivel mundial por la existencia de más de una interrelación económica entre los diferentes países. Las consecuencias se dan también en el ámbito jurídico-legal por el simple razonamiento de que si tú negocias un producto necesariamente esto debe realizarse en un lugar (nacional o internacional) y bajo un instrumento legal cualquiera fuese su origen (contrato). Eso significa que todos los contratos de inversiones entre empresa, estado y consumidores deben realizarse en un determinado país con las garantías que necesita toda persona natural o jurídica que desea realizar sus actividades económicas y qué mejor que realizarlos en un Estado de derecho que es sinónimo de seguridad jurídica.

\section{Hipótesis}

Para afrontar la globalización se requiere de empresas competitivas y de un fuerte Estado de derecho.

\section{ANÁLISIS Y DISCUSIÓN}

La hipótesis planteada nos ha permitido identificar las siguientes variables:

\section{Globalización}

La globalización no es moda (pasará y dará lugar a otra con el tiempo), ni tampoco representa un esquema político. La globalización, como sabemos, es producto del avance tecnológico y de las innovaciones. Entre los instrumentos que han propiciado su gestación tenemos al transporte, la comunicación, el correo electrónico, la homogenización de los métodos de producción, la reducción de las distancias, los tiempos y la simultaneidad de la información a nivel mundial.

Hoy seguimos asistiendo a una internacionalización de la economía (Bueno y otros, 1990:20), después de una crisis financiera global. Está internacionalización se expresa en:

- Se ha acentuado la interdependencia2 entre los países, que se refleja en los flujos internacionales de bienes y servicios, capitales y conocimientos.

- La competitividad es la única manera de garantizar a una empresa su participación activa en la creciente globalización mundial.

- El comercio es cada vez más especializado en productos, por lo que su fabricación tiene una ventaja relativa.

- Los factores explicativos o la intensificación de los intercambios comerciales, así como las relaciones exteriores en general, son de tipo estratégico.

- El mando es cada vez más de plena mutación tecnológica y social.

- El dinamismo que caracteriza a las empresas en la actualidad.

Desde el punto de vista del mercado, la globalización fue creando un impacto a nivel mundial fundamentalmente por la tecnología, con el objetivo de generar en toda la aldea global un gran mercado y que la humanidad pueda producir y consumir cualquier producto en el mundo, así como las relaciones empresariales por medio de las fronteras internacionales con un alcance y complejidad sin precedentes.

El año 2013 ha sido un año complicado para las economías emergentes, en la región Latinoamericana (Klasen, 2012: 33 y 55), pese a ser una región muy heterogénea, ha podido resistir ante el embate financiero. Las diferentes crisis que se dieron históricamente le enseñaron al sector empresarial a prepararse para las eventualidades que 
se pudieran presentar y Perú no estuvo al margen de quienes tuvieron la prudencia y prevención para no ser parte de este proceso de contagio global, cuyas repercusiones en el ámbito empresarial no han sido tan dramáticas como en otros continentes.

\section{Empresa competitiva}

En la actualidad una empresa es un sistema social que crea riqueza y empleo, y que es capaz de adaptarse permanentemente a su entorno. En ese contexto la globalización trajo muchas consecuencias en el sector empresarial, tanto en el entorno nacional como regional, y en el propio entorno interno de la organización.

\section{SITUACIÓN DEL ENTORNO NACIONAL}

La situación nacional en el que se desenvuelve la empresa, ha estado influenciado por muchos aspectos, entre estos podemos citar las siguientes situaciones:

- Se registran, por ahora, precios altos para sus productos primarios.

- Existen flujos de capital para la inversión productiva. Tenemos una creciente competencia global pero existen dificultades a superar: la escasa relevancia en la economía mundial, su insuficiente y costosa infraestructura, insuficiente gasto social, la carencia de adecuadas instituciones civiles, y un sistema policial y judicial lento y no confiable.

- Tenemos un sistema educativo ineficaz, formándose jóvenes con baja productividad en el mercado laboral.

- Valores culturales rentistas poco orientados al proceso de desarrollo del país.

- Si son emprendedores, deben tener claro la estructura legal de la empresa y esto varían según su naturaleza jurídica y su tipo: micro empresa, pequeña empresa, mediana o gran empresa; y de acuerdo al rubro al que pertenezca como industrial, comercial o de servicios.

El Perú necesita una cuidadosa mezcla de protección, subsidios y promoción de las exportaciones como lo demuestran la mayoría de los países que actualmente son ricos y que usaron la venta al exterior para desarrollarse, como Gran Bretaña en el siglo XVII, sin olvidar a Corea y Taiwán en el siglo XX. Los problemas principales y que están pendientes a resolver siguen siendo la integración y reestructuración de la industria peruana y la mejor explotación de nuestros recursos.

La economía peruana, en términos generales, no tiene un déficit alto. Su tasa de ahorro es importante y la inflación está controlada (es casi la tercera parte del promedio regional que se espera sea de un 7\%en el 2014, 32,4\%es el estimado de inflación de Venezuela para el 2014). El país se encuentra en un estado de desarrollo intermedio ya que compite con sus materias primas y busca eficiencia para reducir costos. Algunos creen que pueda seguir sosteniendo altas tasas de crecimiento con la actual infraestructura deficitaria y los progresivos aumentos de dificultades del sector privado para realizar proyectos relacionados a la energía, minería y petróleo, ello debido a los conflictos sociales.

Esta creciente globalización de la economía mundial plantea muchos retos a la vez, y las empresas -entendidas como un sistema social que genera riqueza y empleo y que sea capaz de adaptarse a entornos más amplios y complejos e incértos que son fundamentales para el futuro de sus negocios- ante este escenario nacional, tienen algunos retos que asumir. Entre estos tenemos:

- A nivel interno. En primer lugar, el pensamiento empresarial debe estar acorde a los nuevos tiempos y a las nuevas necesidades; es decir, antes y después de la globalización, rompiendo paradigmas y adaptándose a la globalización. Como parte de estos nuevos vientos la empresa tiene nuevas tareas pendientes a implementar, como:

a) recurso material, es decir el capital financiero y físico. Al financiero nos referimos al dinero necesario para constituir la empresa y desarrollar sus actividades. Al capital físico, que debe estar constituido por bienes o equipos de la empresa como son local, maquinaria, mobiliario, entre otros.

b) el elemento de bienes y servicios, ya que el ingreso de la tecnología afecta a los productos finales que puede ofrecer la empresa que vienen a ser los efectos directos ocasionados por los cambios tecnológicos agregados a los procesos de producción.

c) las nuevas necesidades impuestas por el entorno social donde desarrolla sus productos, así como la parte económica que fija el tipo de producto que el mercado demanda. 
d) En cuanto a la estructura y organización de la empresa, tienen que adaptarse a las nuevas exigencias de producción.

e) En las personas influye la tecnología por cuanto tienen que capacitarse constantemente (formación y perfeccionamiento) (Mantero, 1998: 114), sus hábitos y actitudes de trabajo tienen que adecuarse a los cambios de producción como a los estructurales.

- A nivel externo. Como bien sabemos, toda empresa tiene que ser consciente para enfrentar cambios en su entorno. En el ámbito nacional algunas empresas han estado a punto de quebrar por lo que muchos criticaban que la globalización más que una oportunidad, era una amenaza en el ámbito empresarial, ya que muchos modelos han surgido y caído, otros han sido aceptados y rechazados. En ese contexto podemos decir que las empresas tienen retos que asumir, entre estas citaremos algunas que nos parecen prioritarias, sin desmerecer otras que pudieran existir, y son: a) económico- comercial b) cambios tecnológicos y competitividad, y, c) en la competencia $y, d)$ los consumidores.

\section{Económico - comercial}

Uno de los efectos inmediatos ante este fenómeno global ha sido el surgimiento en algunos continentes - en otros se han fortalecido- las grandes áreas de mercados de integración económico y comercial, como la Unión Europea (UE), el Tratado de Libre Comercio de América del Norte (NAFTA), el Mercado del Cono Sur (Mercosur), la Unión de Naciones de Sudamericanas (UNASUR), la Comunidad Andina (CAN), y últimamente la Alianza del Pacífico (Ugarte Vega-Centeno,2011: 88) incrementando la competencia por los ingresos de productos, así como de bienes y servicios que son recíprocos entre los Estados miembros. La oportunidad que brindan estos mercados hace que las empresas no descarten a corto plazo la posibilidad de exportar directamente, aunque por el momento la mayoría se contenta con cubrir la demanda nacional. Es de mencionar que cuando nos referimos a ganar acceso a nuevos mercados, se trata que los productos no solo deben copar el ámbito nacional sino que deben salir y aprender a competir en el extranjero con valor agregado, y si concretaran las alianzas estratégicas con otras empresas - junto a las nuevas tecnologías- sería mucho mejor en un mercado cada vez más competitivo. La discusión de la propiedad intelectual y la mano de obra calificada son tareas pendientes en el ámbito empresarial.

\section{Tecnología y competitividad}

En un primer momento la tecnología y competitividad jugaron un rol importante en las empresas al permitir un canal de información más rápido gracias al avance de las comunicaciones expresado en el desarrollo de la telefonía celular, el correo electrónico, la prensa, la televisión por cable, y el Internet que viene a ser la piedra angular en la tecnología.

En cambio, hoy podemos decir que la incorporación de la tecnología ha revolucionado el concepto tradicional de mejora de la empresa para ser más eficiente, como el desarrollo de la robótica y del know how. De una parte, con el ingreso de la robótica a las industrias se ha reemplazado a las personas en sus actividades operarias, pero con la robótica la automatización del funcionamiento de las máquinas ha hecho que los recursos humanos (conjunto de personas, empresarios y trabajadores que trabajan en la empresa) realicen trabajos industriales 0 administrativos con poca participación. De otra parte, el know how, junto a la información y los conocimientos, son decisiones fundamentales para tener una empresa competitiva y moderna de acuerdo a los estándares internacionales.

En cuanto a la competitividad, nos referimos a la capacidad de una organización para obtener y mantener ventajas comparativas en el entorno socioeconómico donde se desarrolla, y las empresas peruanas (MITINCl, 1998) creen que todavía no son lo suficientes competitivas para afrontar los cambios de la globalización debido a la falta de planes estratégicos. Sin embargo, la competitividad va de la mano de la empresa y del sector financiero. En el Perú últimamente se tiene mayor acceso al crédito y las mayores facilidades para iniciativas de los negocios empresariales impulsados por el sector financiero que está trayendo mejoras en la competitividad del país.

En el último informe del ranking del Índice de Competitividad Global Mundial (World Economic Forum,2013) Perú subió cinco posiciones y se encuentra en el lugar 40 a nivel mundial en 
desarrollo financiero por su facilidad de acceso a créditos y la solidez del mercado bancario, y entre 48 países evaluados, el Perú es el decimoctavo más seguro para invertir (Instituto de Inversión Black Rock, 2013). Donde se impulsa más inversión privada es en los sectores de turismo, comercio, minería, gastronomía, construcción, educación, salud, carreteras, y otros.

\section{Competencia}

Existen retos a superar en su entorno empresarial, que hace que la empresa comprenda que debe acostumbrarse a trabajar en un ámbito de mayor complejidad estratégica y una progresiva presencia de empresas extranjeras en mercados locales, ya sea por la vía de la exportación como por la inversión directa de países vecinos que compiten en el mercado nacional como es el caso de Ripley, Zaga Falabela, Tottus, Plaza Vea, entre otros.

\section{Los consumidores}

La progresiva tendencia hacia la homogeneización de los gustos y las pautas de comportamiento de los consumidores a través del impacto de la televisión y otros medios transmisores de valores culturales, ha llevado al incremento de productos estándar y globales que permiten a la empresa vender productos en diferentes mercados nacionales e internacionales. Es así como la globalización opera en función de los mercados diferenciados $y$, haciendo uso de las nuevas tecnologías, es posible adaptar los productos a los perfiles de aquellos consumidores (que tienen 206 huesos, 650 músculos, 50 billones de células) y que cada vez son más exigentes. Como consecuencia de estos aspectos, hoy ha surgido el nuevo perfil del consumidor como indican los estudios de mercado que dicen que las personas que empezaron la universidad y nacieron en 1985 en unos pocos años serán consumidores con su propio ingreso.

- Tenían tres años cuando la Unión Soviética se dividió y no recuerdan la "Guerra Fría". Nunca han temido una guerra nuclear. Las letras CCCP son solo una sopa de letras. Ellos solo han conocido una Alemania. En su vida siempre ha existido el SIDA. La expresión "pareces un disco rayado" no significa nada para ellos pues nunca han tenido un tocadiscos. La
"Guerra de las Galaxias" les parece muy irreal y los efectos especiales patéticos. El disco compacto apareció cuando tenían seis años.

- Muchos nunca han tenido una televisión con solo tres canales y menos una televisión en blanco y negro, la mayoría han tenido cable. Siempre han tenido VHS y VCD, pero no tienen idea de qué cosa es Beta. No pueden imaginar qué es no tener un control remoto. Nacieron el año en que Sony puso en el mercado el walkman. Hoy solo conocen el UCB, el MP3 y el MP4.

- Ellos nunca han ido a la playa pensando en la película "Tiburón". El conflicto con el terrorismo de los años 80 y 90 es para ellos una historia antigua como la Primera Guerra Mundial. Y por último, Michael Jackson siempre ha sido blanco.

Este nuevo consumidor es más global, escéptico, analítico, consciente de sus opciones y con menor capacidad de consumo. Es allí donde las empresas tienen que partir para prepararse y afrontar la globalización.

Entonces podemos afirmar que con el acceso a la tecnología y a la información, cambia el rol de las personas como consumidores. Preguntamos a todos Uds. que nos acompañan en esta lectura: ¿hemos cambiado o no de gustos estilo y comportamientos?, ¿tenemos otras necesidades? y así seguiríamos una lista interminable de preguntas.

Cambiamos, sí o no. Pues nada, dejo a Uds. la respuesta, porque ahora ustedes son los nuevos consumidores y contribuyentes, y todo esto nos afecta en muchas formas.

\section{Estado de Derecho}

Las fronteras nacionales ya no son una barrera para las empresas. Los avances tecnológicos y las comunicaciones, principalmente, han revolucionado la forma de realizar negocios. Las empresas no pueden estar al margen ni aisladas de los acontecimientos que vive el mundo que cada vez está más interrelacionado, por lo que es importante tener en consideración las expectativas de la economía sobre los acontecimientos futuros que son de suma importancia porque influyen sobre la conducta de las personas. Ello nos ayudará a entender el devenir de la economía en general y, de esa manera, podremos prever nuestras ganancias e inversiones en una economía de mercado, y qué mejor indicador para la estabilidad y la inversión 
de una empresa que el Estado de Derecho que como consecuencia inmediata le dará seguridad jurídica a todas las personas que deseen realizar sus actividades económicas. Entendemos como Estado de Derecho a aquel país que se rige por un sistema de leyes e instituciones ordenadas en torno a la Constitución y las leyes. El Estado de Derecho se rige por cada una de las leyes que establece la Constitución, y está conformado por diferentes instituciones en las que cada uno cumple sus funciones con autonomía, sin impedimento alguno para que las puedan realizar de acuerdo a ley. Por eso se dice; "En democracia, y con la independencia entre los poderes y con órganos estatales autónomos, el mando tiene que repartirse, y compartirse ejerciéndose dentro de los límites de las competencias de cada poder u órgano, sin que ellos puedan extralimitarse para invadir fueros ajenos" (Ferrero, 2008: 387).

El Estado de Derecho (Atienza, 2011) está relacionado con la globalización porque este fenómeno que interconecta genera interdependencia entre los mercados, organizaciones y sujetos comercialmente, y para ello se necesita de una regulación de éstos, para lo cual se busca que cada uno de los órganos del Estado de Derecho desempeñe debidamente sus competencias y atribuciones. Con el nuevo fenómeno de la globalización algunos ponen en duda el papel del Estado de Derecho debido a que cada uno de los aspectos económicos ha tomado un carácter global, "en este sentido es posible hablar de una economía mundial, es decir, un espacio de circulación unificada" (Durand y otros, 1993: 118). Sin embargo, lejos de desarrollar un papel funcional o subordinado, el Estado de Derecho mantiene un papel crucial y central en la economía mundial que, a pesar de la creciente existencia de flujos y redes globales, la mayo ría de las actividades económicas se dan dentro de las fronteras nacionales y por ello el Estado de Derecho - con las capacidades que le competen- determina el bienestar de la población y de la economía interna.

Todos los fenómenos globales acaban por tener una expresión localizada. La globalización trae como consecuencia jurídica una pluralidad de fuentes de creación del derecho; no obstante, para que éstas sean aplicables en un territorio deben ser reconocidas por los órganos del estado, es decir por el Estado de Derecho, por lo mismo que cada una de las acciones toman nuevas medidas en estos poderes del estado en función de sus competencias que les corresponden y que deberán de ser cumplidas generando un impacto no solo nacional sino internacional como efecto de la globalización.

Así mismo debe reconocerse que las atribuciones de los órganos del estado, en el contexto globalizado, también se han transformado. Existen nuevos órganos internacionales que cumplen funciones al igual que el Estado de Derecho, y sus labores siguen siendo preeminentes pero no exclusivas. "Los efectos de este fenómeno impactan por ello directamente por las funciones que típicamente se han atribuido a los órganos del estado" (López, 2004: 123).

En ese sentido, como resultado de la globalización, el fenómeno jurídico es hoy mucho más complejo y admite una diversidad de fuentes que se generan por mecanismos supra e infra estatales. Ello implica un cambio en la manera de entender el derecho, ya no como un sistema piramidal y territorial sino como un sistema de red en la cual, aunque el estado permanece al centro, no puede más reivindicar un papel único y exclusivo. El crecimiento económico tiene que sustentarse en al menos dos condiciones jurídicas básicas: un marco jurídico transparente que garantice los derechos de propiedad, y un conjunto de instituciones públicas que aseguren eficientemente su protección, en especial los tribunales. Por ello resulta central fortalecer las instituciones responsables de administrar la justicia (López, 2004: 151).

Hoy en día las instituciones del Estado de Derecho son vistas como la clave para el desarrollo económico; actualmente, cuentan con una ola global de reformas que se encaminan hacia una uniformidad de sistemas entre los diferentes países.

En efecto, como sostiene Rodríguez "que la difusión transnacional del Estado de Derecho resulta del choque de dos proyectos globales que surgieron en las últimas décadas del siglo $X X$. De un lado, el neoliberalismo ha enfatizado las reformas institucionales que faciliten el funcionamiento del mercado y preserven el orden público. Del otro lado, el neo constitucionalismo ha insistido en reformas que protejan no solo los derechos civiles y políticos sino también los derechos sociales" (Rodríguez, 2009: 87).

El Estado de Derecho es una lucha contra la arbitrariedad, garantiza la legalidad pública. Ahora 
este Estado de Derecho y la globalización tienen una relación recíproca directa. Los cambios que trae consigo este fenómeno y la potencialidad para conducir a la prosperidad económica demandan la existencia de una gobernanza eficiente y democrática, con instituciones capaces de apoyar y regular el mercado, mecanismos sólidos para erradicar las inequidades sociales y, en especial, suficiente determinación y recursos para atender las necesidades que demanda la sociedad de hoy.

Las constituciones modernas garantizan los derechos económicos de las personas, quienes pueden decidir la actividad económica a la que quieran dedicarse y si invierten deben tener ciertas garantías y seguridades para su inversión, además de realizar con toda libertad su actividad económica. En esa orientación nuestro ordenamiento jurídico peruano respalda cualquier actividad económica de las personas en facultad a la libertad que les concede el artículo 1354 del Código Civil (CC), por el que las personas pueden crear un contrato diferente a los ya regulados para normar sus relaciones. Estos principios son recogidos en el mencionado artículo, que señala al respecto: "Las partes pueden determinar libremente el contenido del contrato, siempre que no sea contrario a norma legal de carácter imperativo". Así mismo, en el siguiente artículo 1355 del CC, se indican las limitaciones en el contrato, al manifestar lo siguiente: "La ley, por consideraciones de interés social, público o ético puede imponer reglas o establecer limitaciones al contenido de los contratos". Esto es concordante con el artículo 62 de la Constitución Política del Perú referente a la libertad de contratación que dispone: "la libertad de contratar garantiza que las partes pueden pactar válidamente según la normas vigentes al tiempo del contrato. Los términos contractuales no pueden ser modificados por leyes u otras disposiciones de cualquier clase. Los conflictos derivados de la relación contractual solo se solucionan en la vía arbitral o en la judicial, según los mecanismos de protección previstos en el contrato o contemplados en la ley. Mediante contratos ley, el Estado puede establecer garantías y otorgar seguridades. No pueden ser modificados legislativamente, sin perjuicio de la protección a que se refiere el párrafo precedente. De acuerdo a estos artículos existen un principio de libertad contractual y autonomía de la voluntad al señalar que las partes pueden determinar libremente el contenido del contrato, siempre que no sea contrario a normas de carácter legal".
Y, por último, existen lecciones a aprehender en cuanto a la crisis internacional para que no vuelva a repetirse y evitemos ser corresponsables de la crisis; para esto es vital tener un mercado fuerte y competitivo y, claro está, se requiere de un estado fuerte y hábil que antes no había porque anteriormente se dejaba todo al mercado para que resuelva por sí mismo los problemas. El estado debe cuidar la relación entre economía y sociedad para evitar la angurria que se produjo en el ámbito financiero en la escena internacional.

No había estado fuerte; al contrario, había ausencia del estado. No había supervisión efectiva, y se hicieron todo tipo de inversiones agresivas sin ningún tipo de respaldo real de producción. Todo esto nos lleva a la reflexión de que lo principal es tener un estado hábil y capaz de intervenir en el momento adecuado para sostener un sistema sólido y confiable.

\section{CONCLUSIONES}

1. El mundo está convirtiéndose cada vez más en una unidad. Las barreras comerciales están cayendo y los mercados se están abriendo. Las empresas se están convirtiendo en globales y las exigencias son otras; por ejemplo, empresas con responsabilidad social

2. El mundo cada vez está más interconectado por el mercado, la moneda, los clientes, la explosión del cable y el Internet, y los nuevos consumidores.

3. En el ámbito empresarial, la toma de decisiones es más rápida; se necesita un liderazgo intrépido y visionario para que la colaboración y confianza en la inversión brindada por un Estado de Derecho sea garantía de la seguridad jurídica.

4. En el Perú, el modelo económico que hemos seguido durante tres go biernos constitucionales (Toledo, García y ahora, Humala) garantizó la independencia del Banco Central de Reserva, se fortaleció la moneda y se expandió la inversión pública y privada. En ese lapso, se incrementó el consumo interno y se abrió la economía al mundo mediante la celebración de los tratados de libre comercio (TLC) y se buscó el posicionamiento de nuevos productos de exportación. El país sigue siendo un nicho de mercado para invertir y se deben proponer mecanismos para hacer más atractiva la inversión con responsabilidad social. Esperemos, 
como dicen la voz popular, que siga la fiesta en el auge económico y que un shock externo no apague la orquesta.

5. Las constituciones modernas garantizan los derechos económicos de todas las personas naturales y jurídicas que deseen realizar cualquier actividad económica siempre y cuando no sea contrario a la ley.

6. Y, por último pensamos que el estado debe cuidar la relación de la economía y la sociedad, ya que un mercado competitivo requiere de un estado fuerte.

\section{LITERATURA CITADA}

Atienza, Manuel. (2011). "Constitucionalismo, globalización y derecho". Editado por Carbonell y García Jaramillo, Comentarios de Ikeda, Jorge En: fuente. [En línea].Fecha de consulta: 02/11/2013. Disponible en:www. jorgeikeda.com/wordpress/? $p=3216$.

Bueno E., y otros. (1990). La empresa española: estructura y resultados. Instituto de Estudios Económicos, Madrid.

Durand, Marie Francoise y otros. (1993). Le monde, espacesetsystemes. Segunda edición. Editorial Presses de la FondationNationale des SciencesPolitiques, París.

Ferrero, Raúl. (2008). La reforma del Estado y la reforma constitucional pendiente. Editorial Grijley, Lima.

Instituto de Inversión Black Rock. (2013). Perú supera a otros países en mayor seguridad para invertir, en Perú noticias, disponible en: perunoticias.net/tag/instituto-inversionblackrock.

Kenichi, Ohmae. (1992). El mundo sin fronteras. Editorial Mc Graw Hill, México.

Klasen, Stephan. (2012). "El impacto de la globalización sobre el desarrollo económico y social en América Latina". En: Bien Común.
Año 17, N²02 México en el Mundo. Octubre del 2011. Fundación Rafael Preciado, México. Fecha de consulta: 06/11/2013. Disponible en: < http://www.fundacionpreciado. org.mx/biencomun/bc202/S_Klasen.pdf > .

López Ayllón, Sergio. (2004). “Globalización, Estado de Derecho y Seguridad Jurídica".Corte Suprema de Justicia de la Nación, México. [En línea].Fecha de consulta: 20/08/2013. Disponible en: < http://biblio.juridicas. unam.mx/libros/libro.htm?|=1572> .

Mantero de San Vicente, Osvaldo. (1998). "El derecho del trabajo ante la globalización de la economía". En: Globalización económica y negociación colectiva. Regimen financiero y administrativo de la Seguridad Social. Ponencia presentada en el IV Congreso Regional Americano de Derecho del Trabajo y de la Seguridad Social. Tomo 2. Sociedad Chilena de Derecho del Trabajo y de la Seguridad Social, Santiago de Chile.

MITINCI. (1998). IV Cumbre de la competitividad, conocimiento y liderazgo empresarial. Lima, octubre de 1998.

Rodríguez, César. (2009). La Globalización del Estado de Derecho. Editorial Universidad de los Andes - Centro de Investigaciones Sociojurídicas, Colombia.

Ugarte Vega Centeno, Máximo. (2011). "La globalización y los círculos concéntricos de integración en el nuevo orden económico mundial". En: Gestión en el Tercer Milenio. Revista de Investigación de la Facultad de Ciencias Administrativas de la Universidad Nacional Mayor de San Marcos. N²7. Julio del 2011. Lima, Facultad de Ciencias Administrativas de la Universidad Nacional Mayor de San Marcos.

World Economic Forum.(2013). Informe Global de Competitividad 2012-2013.World Economic Forum, Geneva. 